\title{
THIẾT KẾ CHẾ TẠO Hệ THỐNG QUÉT ĐỊA HÌNH MẶT ĐẤT DỰA TRÊN CÔNG NGHỆ ẢNH SỐ THU NHẬN DŨ LIỆ THÔNG TIN ĐỊA LÝ VÀ THÀNH LẬP BẢN ĐỔ ĐỊA HİNH
}

\author{
ĐẬNG XUÂN THỦY(1), LUUU HẢI ÂU ${ }^{(1)}$, NGÔ TH!̣ LIÊN ${ }^{(1)}$, LUUU HẢI BẰNG $^{(2)}$ \\ (l) Viện Khoa hoc Đo đạc và Bản đồ \\ (2)Viện Khoa học và Công nghệ Giao thông vận tải
}

\section{Tóm tắt:}

Hiện nay, việc ưng dụng công nghệ ảnh số trong thu thập dũ liệu thông tin địa lý và thành lập bản đồ địa hình đã trở lên phổ biến trên thế giới và ở Việt Nam nhờ nhũng ưu điểm của công nghệ này nhu giảm thời gian thi công, giảm nhân công lao động qua đó mang lại lợi ich kinh tế cao. Được đánh giá có khả năng úng dụng lớn tuy nhiên công nghệ ảnh số thường phải kết hợp với việc đo vẽ trục tiếp trong quy trình thu thập dũ liệu thông tin địa lý và thành lập bản đồ địa hình do nhũng hạn chế chua thể khắc phục được trong thực tế nhu: thiếu dũ liệu địa hình và địa vật tại nhũng khu vực có độ che phủ cao, hạn chế trong thu thập dũ liệu chiều đứng của địa vật... Do đó, để hoàn thiện quy trình úng dụng công nghệ ảnh số trong thu thập dũ liệu thông tin địa lý và thành lập bản đồ địa hình, cần có nhũng nghiên cưu để khắc phục những nhược điểm trên. Nhóm tác giả tiến hành nghiên cưu theo định hướng áp dụng công nghệ đo ảnh mặt đất để khắc phục nhũng nhược điểm trên. Bài báo này giới thiệu hệ thống quét địa hình mặt đất dựa trên công nghệ ảnh số được tích hợp bởi camera ảnh số, máy định vị GNSS và một thiết bị IMU. Bên cạnh đó, bài báo tiến hành khảo sát độ chính xác của hệ thống này trong thu nhận dũ liệu thông tin địa lý, thành lập bản đồ địa hình.

\section{1. Đặt vấn đề}

Hiện nay, việc thành lập bản đồ địa hình và thu thập dữ liệu không gian địa lý đang được thực hiện bởi nhiều công nghệ và thiết bị hiện đại. Phương pháp đo đạc trực tiếp sử dụng các loại máy toàn đạc điện tử, máy GPS hay hiện đại hơn là máy quét laser mặt đất và công nghệ bay quét Lidar. Phương pháp sử dụng công nghệ ảnh số bao gồm đo đạc ảnh hàng không, ảnh UAV và ảnh chụp mặt đất. Các giải pháp trên đã cơ bản giải quyết được yêu cầu thành lập bản đồ và thu thập dữ liệu thông tin địa lý. Tuy nhiên các thiết bị đang sử dụng được nhập khẩu hoàn toàn từ nước ngoài với chi phí cao.

Khái niệm hệ thống quét địa hình mặt đất ở đây được hiểu là một khối chặt chẽ được tích hợp bởi các thiết bị bao gồm camera ảnh số, thiết bị GNSS và IMU có thể ghi nhận hình ảnh xung quanh một cách liên tục trên một phạm vi rộng.

Mô hình số địa hình với độ phân giải cao và độ chính xác cao có thể cung cấp dữ liệu để phát hiện những sự thay đổi về độ cao phục vụ cho một số mục đích như xói mòn hoặc quan trắc các dịch chuyển liên tục của địa hình [1]. Trong công tác thành lập bản đồ địa hình tỷ lệ lớn ở những khu vực có sự thay đổi phức tạp về độ cao thì các phương pháp đo đạc truyền thống không đảm bảo được mật độ cũng như khả năng thu thập dữ liệu mất nhiều thời gian và công sức. Phương pháp bay chụp UAV có khả năng thu thập dữ liệu nhanh nhưng không hiệu quả trong một khu vực nhỏ và yêu cầu phát hiện những thay đổi rất nhỏ về địa hình, hay những khu vực có độ che phủ cao và độ chính xác về độ cao của phương pháp bay chụp ảnh UAV kém hơn so với các phương pháp khác. Các hệ thống máy quét laser có thể 
đáp ứng yêu cầu thu thập dữ liệu này. Mặc dù mô hình $3 \mathrm{D}$ được tạo ra từ công nghệ quét laser chứa một lượng lớn các điểm chi tiết, nhưng vẫn khó có thể xác định các vết nứt hoặc những thay đổi rất nhỏ của bề mặt. Một số nghiên cứu đã chỉ ra rằng việc kết hợp công nghệ quét laser với chụp ảnh là cần thiết và sẽ mang lại hiệu quả cao hơn [2][3]. Một số thiết bị quét laser hiện nay đã trang bị cả tính năng chụp ảnh. Tuy nhiên, các thiết bị laser hiện nay có giá thành rất cao do vậy khả năng tiếp cận trong một số công việc khảo sát bị hạn chế. Nghiên cứu [4] đã cho thấy việc sử dụng kỹ thuật cấu trúc từ chuyển động (Structure-from-Motion - Sfm) có thể dựng lại mô hình $3 \mathrm{D}$ từ ảnh chụp mặt đất và đạt độ chính xác tới ( \pm 0.1 mét hoặc nhỏ hơn) khi so sánh mô hình DEM được tạo ra bởi kỹ thuật Sfm với một mô hình tương tự sử dụng phương pháp quét laser mặt đất.

Trong bài báo này tác giả tiến hành giới thiệu và đánh giá độ chính xác của hệ thống quét địa hình mặt đất do nhóm tác giả tự phát triển. Hệ thống thiết bị này có khả năng thu thập dữ liệu thông tin địa lý dựa trên công nghệ ảnh số chi tiết và nhanh chóng với chi phí thấp hơn so với các loại máy quét laser đắt tiền.

\section{Giới thiệu hệ thống quét địa hình mặt} đất

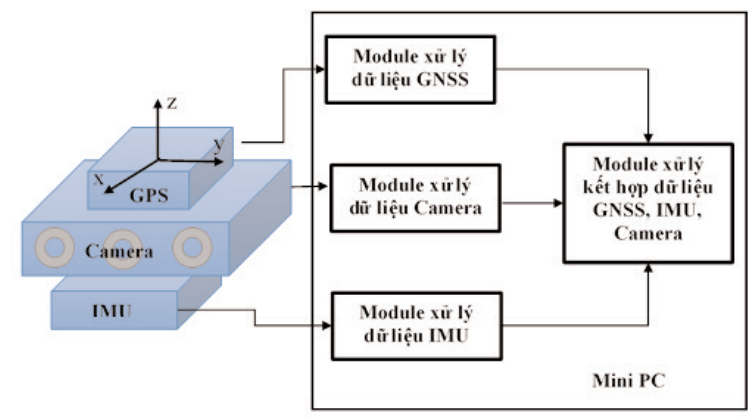

Hình 1: Mô hình tích hợp hệ thống quét địa hìn mặt đất
Thiết bị quét địa hình mặt đất được cấu tạo bởi 4 thành phần chính bao gồm: 3 camera ảnh số, thiết bị GNSS, thiết bị IMU và một mini PC với các thông số kỹ thuật như sau:

\subsection{Giới thiệu thông số thiết bị IMU}

Thiết bị IMU là loại cảm biến PMU 6050 với các thông số:

- Điện áp sử dụng: 3 5VDC

- Điện áp giao tiếp: 3 5VDC

- Chuẩn giao tiếp: I2C

- Giá trị Gyroscopes trong khoảng: +/- 250 50010002000 degree/sec

- Giá trị Acceleration trong khoảng: $+/-2 \mathrm{~g}$, $+/-4 \mathrm{~g},+/-8 \mathrm{~g},+/-16 \mathrm{~g}$

2.2. Giới thiệu thông số thiết bị định vị GNSS. (Xem bảng 1)

\subsection{Giới thiệu thông số thiết bị các Camera}

(Xem bảng 2)

\subsection{Giới thiệu thiết bị Mini PC}

- Cpu: Intel Atom

- RAM: 2GB

- Bộ nhớ: 32GB ổ lưu trữ tích hợp

- Kết nối giao tiếp: Wi-Fi 802.11ac dải kép, Bluetooth 4.1, các cổng USB 3.0 và USB 2.0, một đầu cắm âm thanh, và một cổng HDM

Khi thiết bị được lắp đặt trên các vật mang như ô tô và xe tự hành thì vị trí lắp đặt như trên Hình 2. 

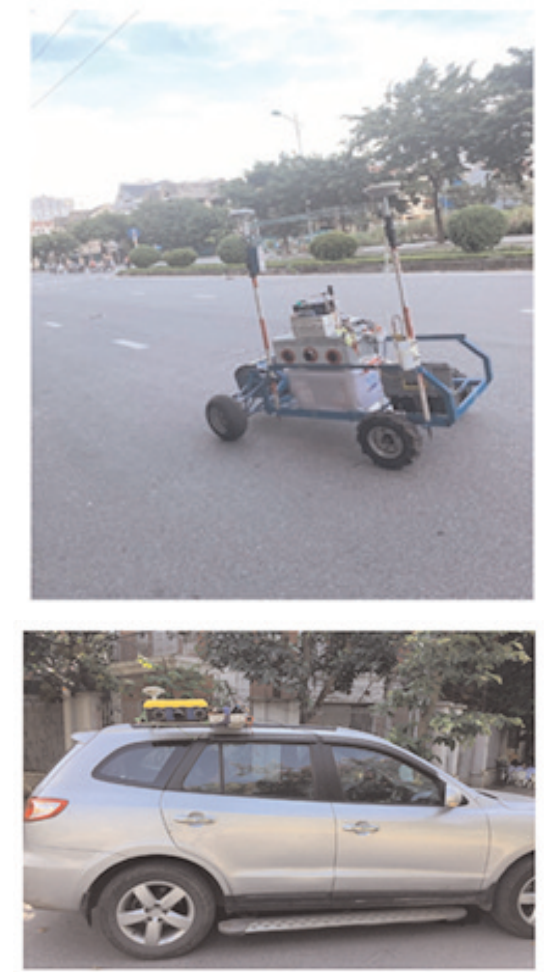

Hình 2: Hệ thống quét địa hình mặt đất gắn trên xe tư hành và ô tô

3. Kết quả thử nghiệm đo đạc bằng hệ thống quét địa hình mặt đất

\subsection{Bố trí bãi thử nghiệm}

Bãi hiệu chuẩn được lựa chọn trên địa hình tương đối bằng phẳng, có các đối tượng địa hình địa vật đặc trưng để phục vụ công tác kiểm tra, đánh giá. Tại khu vực bãi thử nghiệm đánh dấu 45 điểm bao gồm các điểm khống chế ảnh và điểm kiểm tra sau này. Các điểm khống chế và điểm kiểm tra được đo đạc chi tiết theo hệ tọa độ quốc gia VN-2000 và hệ độ cao Nhà nước (Hòn Dấu). (Xem hình 3, 4, 5, bảng 3)

\subsection{Xác định hệ số hiệu chỉnh của camera}

Các bước xác định số hiệu chỉnh camera được tiến hành như sau:
Bước 1: Xử lý dữ liệu ảnh bằng phần mềm Pix4D hoặc Agisoft Photoscan sử dụng toàn bộ khống chế ảnh để xác định tọa độ tâm ảnh chính xác. (tiến hành với 2 ca chụp ảnh $\mathrm{Ca}$ số 1 và $\mathrm{Ca}$ số 2).

Bước 2: So sánh tọa độ tâm ảnh sau xử lý và tọa độ tâm ảnh đồng bộ từ dữ liệu GNSS/IMU và xác định số hiệu chỉnh của camera.

Nhận xét: Dựa vào đồ thị biểu diễn sự biến thiên của sai số các giá trị tọa độ và độ cao của tâm ảnh qua các ca chụp khác nhau ta nhận thấy: Các sai số biến thiên có hệ thống theo từng camera và các giá trị này tương đối đều nhau giữa các ca chụp, thể hiện độ lệch của tâm camera với tâm GNSS và IMU. (Xem hình 6)

Qua tính toán giá trị trung bình giữa các ca chụp thu được bảng số hiệu chỉnh của các camera như sau: (Xem bảng 4)

Các giá trị hiệu chỉnh được sử dụng cho ca chụp tiếp theo để kiểm tra và đánh giá độ chính xác của thiết bị.

\section{3. Đánh giá kết quả}

Sử dụng số hiệu chỉnh để tính toán lại tọa độ tâm ảnh của ca chụp số 3 , sau xử lý tiến hành so sánh giá trị tọa độ của các điểm kiểm tra thu được kết quả như sau: (Xem bảng 5)

Kết quả so sánh giá trị tọa độ của các điểm kiểm tra cho thấy:

$\mathrm{M}_{\mathrm{h}}= \pm 0.042 \mathrm{~m} ; \mathrm{M}_{\mathrm{STB}}= \pm 0.057 \mathrm{~m}$

Trong đó: $\mathrm{M}_{\mathrm{h}}$ là sai số độ cao trung bình.

$\mathrm{M}_{\mathrm{STB}}$ là sai số vị trí mặt phẳng trung bình.

Độ chính xác về mặt phẳng và độ cao đạt độ 
Bảng 1: Thông số thiết bị SQ-GNSS

\begin{tabular}{|c|c|c|}
\hline TT & & hông số kỹ thuật \\
\hline 1 & Số lượng kênh thu đồng thời & 192 kênh \\
\hline 2 & Hệ thống vệ tinh định vị & GPS L1+L2, Glonass G1, Beidou B1+B3 \\
\hline 3 & Công nghệ cải chính RTK & Có hỗ trợ, đạt sai số đến centimet \\
\hline 4 & Tần số xuất tọa độ & Tối đa $10 \mathrm{~Hz}$ (10 vị trí trong 1 giây) \\
\hline 5 & Độ chính xác khi đo độc lập & Phương ngang: $1,5 \mathrm{~m}$, phương đứng $3,0 \mathrm{~m}$ \\
\hline 6 & Độ chính xác PPK & P.ngang: $10 \mathrm{~mm} \pm 1 \mathrm{ppm}$, P.đứng: $15 \mathrm{~mm} \pm 1 \mathrm{ppm}$ \\
\hline 7 & Độ chính xác khi di chuyển & $0,03 \mathrm{~m} / \mathrm{s}$ \\
\hline 8 & Thời gian xác định tọa độ lần đầu & Nhỏ hơn 50 giây \\
\hline 9 & Thừi gian khởi động & Nhỏ hơn 10 giây \\
\hline 10 & Cổng giao tiếp & Cồng COM DB9-RS232, Bluetooth \\
\hline 11 & Nguồn điện & $5 \mathrm{~V}$ \\
\hline 12 & Công suát & $2.5 \mathrm{~W}$ \\
\hline
\end{tabular}

Bảng 2: Bảng thông số thiết bị camera

\begin{tabular}{|c|c|c|c|}
\hline STT & Nội dung & $\begin{array}{c}\text { Thông số kỹ thuật camera Sony } \\
\text { ILCE } 5100\end{array}$ & $\begin{array}{c}\text { Thông số kỹ thuật camera Sony } \\
\text { ILCE } 6000\end{array}$ \\
\hline 1 & Tống số điểm ành & Xấp xi 24.7 triệu điểm ành & Khoảng 24.7 MP \\
\hline 2 & Càm biến hình ành & $\begin{array}{l}\text { Cảm biến APS-C }(23.5 \times 15.6 \mathrm{~mm}) \text {, } \\
\text { Exmor CMOS }\end{array}$ & Loại APS-C $(23,5 \times 15,6 \mathrm{~mm})$ \\
\hline 3 & Định dạng ghi hình & $\begin{array}{l}\text { JPEG (DCF Ver. 2.0, Exif Ver.2.3, } \\
\text { MPF Baseline), RAW (Sony ARW } \\
2.3 \text { ) }\end{array}$ & $\begin{array}{l}\text { JPEG (DCF Ver. 2.0, Exif } \\
\text { Ver.2.3, MPF Baseline), RAW } \\
\text { (Sony ARW 2.3) }\end{array}$ \\
\hline 4 & $\begin{array}{l}\text { Kich thước ành } \\
\text { chụp }\end{array}$ & $\begin{array}{l}\text { Khi chupp tỹ lệ } 16: 9: \\
\text { L: } 6000 \text { x } 3376(20 \mathrm{M}), \mathrm{M}: 4240 \mathrm{x} \\
2400(10 \mathrm{M}), \mathrm{S}: 3008 \times 1688(5.1 \mathrm{M}) \\
\text { Khi chup tỹ lẹ } 3: 2: \\
\text { L: } 6000 \times 4000(24 \mathrm{M}), \mathrm{M}: 4240 \mathrm{x} \\
2832(12 \mathrm{M}), \mathrm{S}: 3008 \times 2000(6.0 \mathrm{M})\end{array}$ & $\begin{array}{l}\text { Khi chụp tỷ lệ 16:9: } \\
\text { L: } 6000 \times 3376(20 \mathrm{M}), \mathrm{M}: 4240 \mathrm{x} \\
2400(10 \mathrm{M}), \mathrm{S}: 3008 \mathrm{x} \\
\text { 1688(5.1M) } \\
\text { Khi chụp ty } 1 \mathrm{e} 3: 2: \\
\text { L: } 6000 \times 4000(24 \mathrm{M}), \mathrm{M}: 4240 \mathrm{x} \\
2832(12 \mathrm{M}), \quad \text { S: } 3008 \mathrm{x} \\
2000(6.0 \mathrm{M})\end{array}$ \\
\hline 5 & $\begin{array}{l}\text { Số lượng khung } \\
\text { hinh có thể ghi }\end{array}$ & $\begin{array}{l}\text { JPEG Chất lượng cao L: } 56 \text { khung } \\
\text { hình, JPEG Tiêu chuẩn L: } 75 \text { khung } \\
\text { hình, RAW: } 23 \text { khung hình, RAW \& } \\
\text { JPG: } 22 \text { khung hình }\end{array}$ & $\begin{array}{l}\text { JPEG Chất lượng cao L: } 56 \\
\text { khung hinh, JPEG Tiêu chuẩn L: } \\
75 \text { khung hình, RAW: } 23 \text { khung } \\
\text { hinh, RAW \& JPG: } 22 \text { khung } \\
\text { hinh }\end{array}$ \\
\hline 6 & Tốc độ chụp & $\begin{array}{l}\text { Chụp liên tục: Hi: tối đa } 6 \text { fps, Lo: } \\
\text { tối đa } 3 \text { fps }\end{array}$ & $\begin{array}{l}\text { Tốc độ ổ đĩa liên tục (xấp xi tối đa) } \\
11 \text { hinh/giây (ơ chể độ chụp liền tục) }\end{array}$ \\
\hline 7 & Nguồn tiêu thụ & $\begin{array}{l}\text { Ánh tĩnh: xấp xi } 2.3 W \text { (vơi ống kính } \\
\text { theo máy E PZ } 16-50 \mathrm{~mm} \text { F3.5-5.6 } \\
\text { OSS) }\end{array}$ & $\begin{array}{l}\text { Thời lượng pin (Ảnh tĩnh): Lên } \\
\text { đến } 360 \text { anh } \\
\text { Sạc pin bên trong: Có }\end{array}$ \\
\hline 8 & Trọng lượng & $\begin{array}{l}\text { Thân máy: } \approx 224 \text { gram } \\
\text { Bao gồm Pin và thẻ nhớ } \approx 283 \text { gram }\end{array}$ & $\begin{array}{l}\text { Trọng lượng (bao gồm pin và thè } \\
\text { nhớ) } \\
285 \mathrm{~g} \text { (Chi } 3 \text { han máy) } / 344 \mathrm{~g} \\
\text { (Kèm pin và phương tiện) }\end{array}$ \\
\hline 9 & Kích thước & $\approx 109.6 \times 62.8 \times 35.7 \mathrm{~mm}$ & $\begin{array}{l}\text { Kích thước (D x R x C) } \\
120 \times 66,9 \times 45,1 \mathrm{~mm}\end{array}$ \\
\hline
\end{tabular}



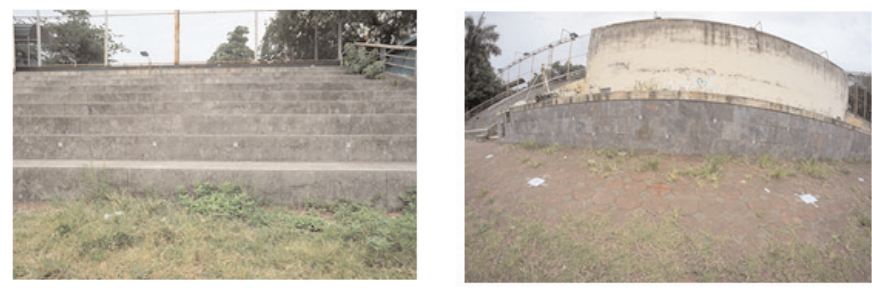

Hìn 3: Khu vục bãi thủ nghiệm

Bảng 3: Bảng giá trị tọa độ các điểm khống chế và điểm kiểm tra

\begin{tabular}{|c|c|c|c|c|c|c|c|c|c|}
\hline \multirow{2}{*}{$S \pi T$} & \multirow{2}{*}{$\begin{array}{l}\text { Tên } \\
\text { điểm }\end{array}$} & \multicolumn{3}{|c|}{ Tọa độ đo đạc thực địa } & \multirow[t]{2}{*}{ STT } & \multirow{2}{*}{$\begin{array}{l}\text { Tên } \\
\text { điểm }\end{array}$} & \multicolumn{3}{|c|}{ Tọa độ đo đạc thực địa } \\
\hline & & $y(m)$ & $x(m)$ & $h(m)$ & & & $y(m)$ & $x(m)$ & $h(m)$ \\
\hline 1 & KT1 & 582408.600 & 2327968.391 & 7.310 & 24 & KT32 & 582407.671 & 2327947.634 & 7.230 \\
\hline 2 & $\mathrm{KT} 2$ & 582408.630 & 2327967.099 & 7.357 & 25 & KT33 & 582407.692 & 2327947.032 & 6.897 \\
\hline 3 & $\mathrm{KC3}$ & 582408.630 & 2327965.644 & 7.292 & 26 & KT34 & 582407.706 & 2327946.129 & 7.200 \\
\hline 4 & KT4 & 582408.642 & 2327964.550 & 7.263 & 27 & KT35 & 582407.703 & 2327945.366 & 6.924 \\
\hline 5 & KT5 & 582408.682 & 2327963.093 & 7.343 & 28 & KT37 & 582407.730 & 2327943.308 & 6.933 \\
\hline 6 & KT9 & 582408.669 & 2327962.001 & 7.257 & 29 & KT38 & 582407.746 & 2327942.209 & 7.231 \\
\hline 7 & KT10 & 582408.690 & 2327960.610 & 7.328 & 30 & KT39 & 582407.748 & 2327941.427 & 6.917 \\
\hline 8 & $\mathrm{KT} 12$ & 582408.723 & 2327959.399 & 7.274 & 31 & $\mathrm{KT} 40$ & 582407.770 & 2327940.362 & 7.216 \\
\hline 9 & KT11 & 582408.714 & 2327958.001 & 7.346 & 32 & KT41 & 582407.726 & 2327939.353 & 6.906 \\
\hline 10 & KT16 & 582407.854 & 2327956.836 & 7.020 & 33 & $\mathrm{KT} 42$ & 582407.808 & 2327938.017 & 7.188 \\
\hline 11 & KT17 & 582407.682 & 2327955.162 & 7.234 & 34 & KT43 & 582407.748 & 2327936.974 & 6.927 \\
\hline 12 & KT27 & 582407.672 & 2327954.543 & 6.961 & 35 & KT44 & 582407.713 & 2327935.559 & 7.215 \\
\hline 13 & KT18 & 582407.684 & 2327954.119 & 7.231 & 36 & KT45 & 582407.710 & 2327934.475 & 6.917 \\
\hline 14 & KT36 & 582407.707 & 2327944.315 & 7.202 & 37 & KT46 & 582407.779 & 2327933.652 & 7.204 \\
\hline 15 & KT50 & 582408.896 & 2327927.338 & 7.264 & 38 & KT57 & 582408.701 & 2327932.971 & 7.200 \\
\hline 16 & KT56 & 582408.874 & 2327920.882 & 7.360 & 39 & KT47 & 582408.903 & 2327931.325 & 7.221 \\
\hline 17 & KT21 & 582407.673 & 2327952.309 & 6.945 & 40 & KT48 & 582408.796 & 2327929.819 & 7.304 \\
\hline 18 & $\mathrm{KT} 25$ & 582407.668 & 2327951.683 & 7.247 & 41 & KT49 & 582408.773 & 2327928.679 & 7.275 \\
\hline 19 & KT26 & 582407.662 & 2327951.194 & 6.913 & 42 & KT51 & 582408.778 & 2327926.205 & 7.277 \\
\hline 20 & KT28 & 582407.652 & 2327950.339 & 7.219 & 43 & KT52 & 582408.864 & 2327924.879 & 7.279 \\
\hline 21 & КT29 & 582407.683 & 2327949.921 & 6.959 & 44 & $\mathrm{KT} 53$ & 582408.787 & 2327923.788 & 7.298 \\
\hline 22 & KT30 & 582407.665 & 2327949.056 & 7.195 & 45 & KT54 & 582408.876 & 2327922.403 & 7.305 \\
\hline 23 & KT31 & 582407.692 & 2327948.392 & 6.921 & & & & & \\
\hline
\end{tabular}

chính xác cho đo vẽ bản đồ địa hình 1:500 với khoảng cao đều 0.5 mét (Theo Thông tu số 68/TT-BTNMT ngày 22/12/2015 Quy định kĩ thuật đo đạc trục tiếp địa hình phuc vu thành lập bản đồ địa hình và co sở dũ liệu nền địa lý tý lệ 1:500, 1:1.000, 1:2.000, 1:5.000).

\section{Kết luận}

Qua quá trình nghiên cứu và thực nghiệm tác giả nhận thấy, hệ thống quét địa hình mặt đất dựa trên công nghệ ảnh số đáp ứng được yêu cầu kỹ thuật thành lập các loại bản đồ địa hình tỷ lệ lớn đến 1:500 thậm chí các loại bản đồ tỷ lệ lớn hơn. Ngoài ra, có thể tăng cường thêm cho các sản phẩm bản đồ nền địa lý một các trực quan và chi tiết hơn.

Trong thực tế, nếu so sánh khả năng thu nhận dữ liệu nhanh chóng và trên một phạm vi lớn hơn thì công nghệ bay chụp ảnh UAV hoàn toàn chiếm ưu thế. Tuy nhiên, đối với các khu vực 


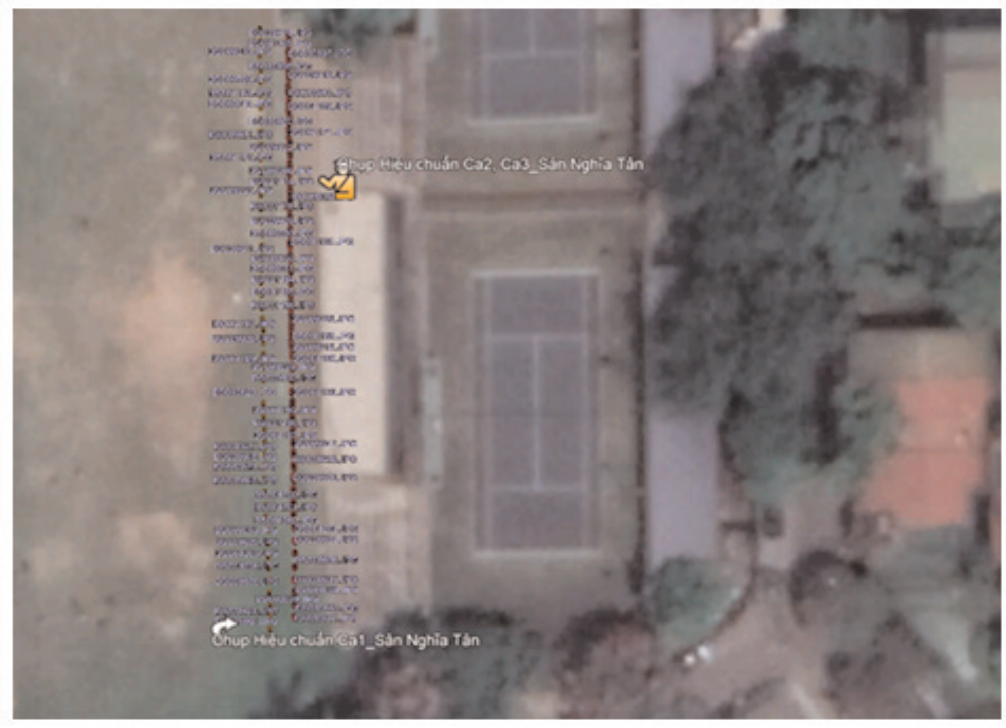

Hình 4: Tọa độ tâm ảnh các ca chụp thử nghiệm

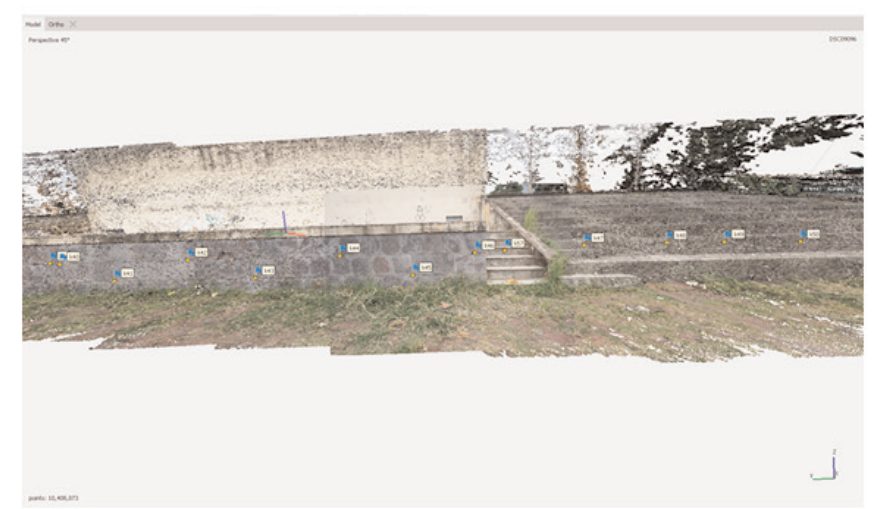

Hình 5: So đồ bố trí các điểm khống chế và điểm kiểm tra

đông dân cư, nhà cao tầng dày đặc, hay khu vực mật độ che phủ cao, hoặc các khu vực không thể sử dụng máy bay không người lái thì việc sử dụng thiết bị quét địa hình mặt đất lại rất hữu ích và mang lại hiệu quả cao trong công tác thu thập dữ liệu thông tin địa lý. Do vậy, việc sử dụng kết hợp công nghệ bay chụp ảnh UAV và máy chụp ảnh mặt đất trong thành lập bản đồ địa hình có thể bổ sung khắc phục những ưu nhược điểm của nhau, tiến tới tự động hóa hoàn toàn công tác thành lập bản đồ địa hình độ chính xác cao.

Đây là nền tảng để tiến tới tự chủ sản xuất thiết bị Mobile mapping dựa trên công nghệ ảnh số để thành lập bản đồ địa hình tỷ lệ lớn và thu thập dữ liệu thông tin địa lý độ chính xác cao. $\bigcirc$

\section{Tài liệu tham khảo}

[1]. James, M.R., Robson, S., 2012. Straightforward reconstruction of 3D surfaces and topography with a camera: accuracy and 


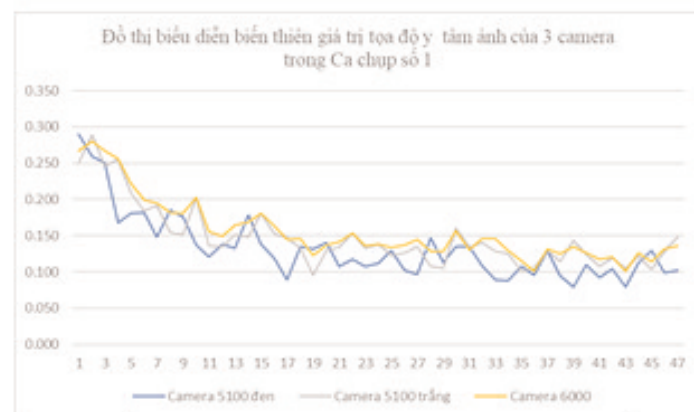

a1) Biển thiên gia trị tọa độ y của tâm ảnh ca chụp số 1

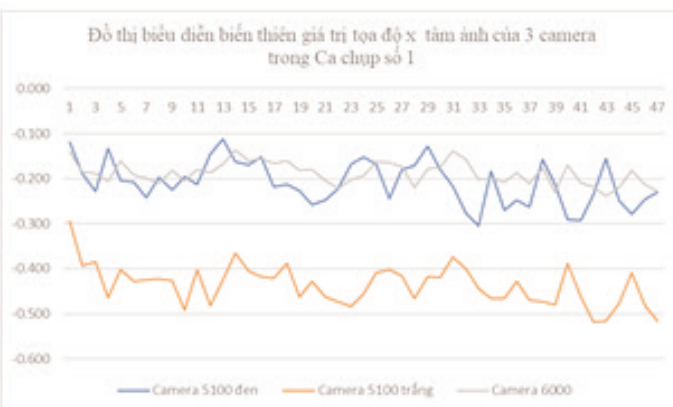

b1) Biển thiên giá trị tọa độ $\mathrm{x}$ của tâm ảnh ca chụp sô 1

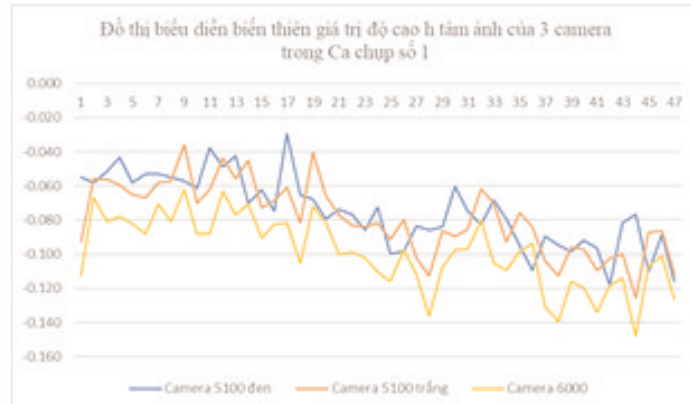

c1) Biển thiên giả trị độ cao $\mathrm{h}$ của tâm ảnh ca chụp sồ 1

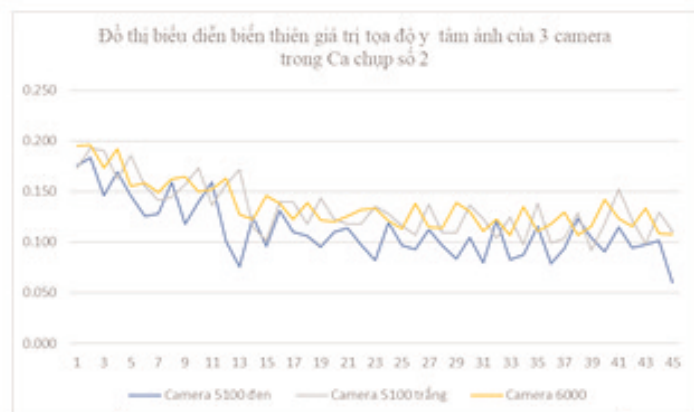

a2) Biển thiên giả trị tọa độ y của tâm ảnh ca chụp sổ 2

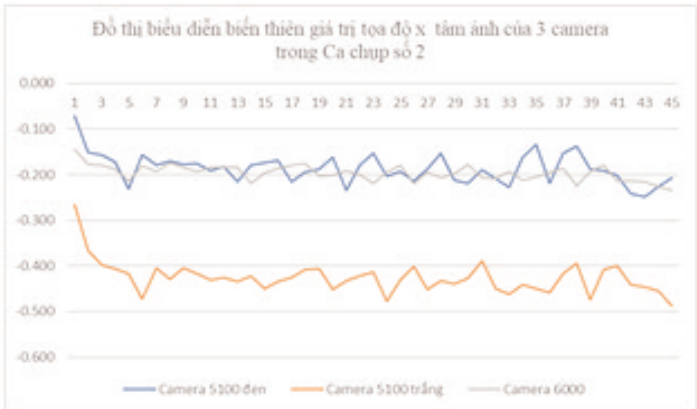

b2) Biển thiên giá trị tọa độ $\mathrm{x}$ của tâm ảnh ca chụp sồ 2

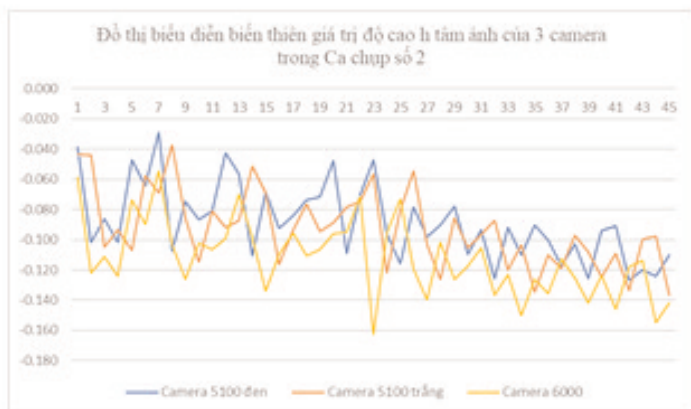

c2) Biền thiên giá trị độ cao $\mathrm{h}$ của tâm ảnh ca chụp sổ 2

Hình 6: Đồ thị biểu diễn biến thiên sai số tọa độ tâm ảnh của 2 ca chụp

Bảng 4

\begin{tabular}{|c|c|c|c|c|c|c|c|c|}
\hline \multirow{2}{*}{$\mathrm{TT}$} & \multirow{2}{*}{ Tên camera } & \multirow{2}{*}{$\begin{array}{c}\text { Ca chụp } \\
\text { số }\end{array}$} & \multicolumn{3}{|c|}{ Giá trị hai lần chụp } & \multicolumn{3}{|c|}{ Giá tri trung bình } \\
\hline & & & $\operatorname{deta} x$ & delta $y$ & delta $\mathrm{h}$ & delta $\mathrm{x}$ & delta y & delta $\mathrm{h}$ \\
\hline \multirow{2}{*}{1} & \multirow{2}{*}{$\begin{array}{c}\text { Sony ILCE } \\
5100 \text { den }\end{array}$} & $\mathrm{Ca} 1$ & 0.132 & -0.208 & -0.075 & \multirow{2}{*}{0.122} & \multirow{2}{*}{-0.197} & \multirow{2}{*}{-0.082} \\
\hline & & $\mathrm{Ca} 2$ & 0.112 & -0.187 & -0.089 & & & \\
\hline \multirow{2}{*}{2} & \multirow{2}{*}{$\begin{array}{l}\text { Sony ILCE } \\
5100 \text { trắng }\end{array}$} & $\mathrm{Ca} 1$ & 0.146 & -0.437 & -0.080 & \multirow{2}{*}{0.139} & \multirow{2}{*}{-0.433} & \multirow{2}{*}{-0.087} \\
\hline & & $\mathrm{Ca} 2$ & 0.131 & -0.429 & -0.095 & & & \\
\hline \multirow{2}{*}{3} & \multirow{2}{*}{ Sony A6000 } & $\mathrm{Ca} 1$ & 0.154 & -0.189 & -0.100 & \multirow{2}{*}{0.144} & \multirow{2}{*}{-0.193} & \multirow{2}{*}{-0.107} \\
\hline & & $\mathrm{Ca} 2$ & 0.134 & -0.197 & -0.114 & & & \\
\hline
\end{tabular}


Bảng 5: So sánh độ chính xác của các điểm kiểm tra

\begin{tabular}{|c|c|c|c|c|c|c|c|c|c|}
\hline \multirow{2}{*}{$\begin{array}{c}\text { Tên } \\
\text { điểm }\end{array}$} & \multicolumn{3}{|c|}{ Tọa độ đo đạc thực địa } & \multicolumn{3}{|c|}{ Tọa độ xác định từ mô hình } & \multicolumn{3}{|c|}{ Sai số } \\
\hline & $y(m)$ & $x(m)$ & $\mathrm{h}(\mathrm{m})$ & $y(m)$ & $x(\mathrm{~m})$ & $h(m)$ & $y$-error & x-error & $z$-error \\
\hline $\mathrm{KT} 1$ & 582408.600 & 2327968.391 & 7.310 & 582408.615 & 2327968.376 & 7.297 & 0.015 & -0.015 & -0.013 \\
\hline $\mathrm{KT} 2$ & 582408.630 & 2327967.099 & 7.357 & 582408.635 & 2327967.130 & 7.315 & 0.005 & 0.031 & -0.042 \\
\hline $\mathrm{KT} 4$ & 582408.642 & 2327964.550 & 7.263 & 582408.652 & 2327964.564 & 7.306 & 0.010 & 0.014 & 0.043 \\
\hline KT5 & 582408.682 & 2327963.093 & 7.343 & 582408.664 & 2327963.098 & 7.306 & -0.018 & 0.005 & -0.037 \\
\hline КT9 & 582408.669 & 2327962.001 & 7.257 & 582408.676 & 2327962.002 & 7.295 & 0.007 & 0.001 & 0.038 \\
\hline KT10 & 582408.690 & 2327960.610 & 7.328 & 582408.691 & 2327960.623 & 7.304 & 0.001 & 0.013 & -0.024 \\
\hline KT12 & 582408.723 & 2327959.399 & 7.274 & 582408.701 & 2327959.435 & 7.310 & -0.022 & 0.036 & 0.036 \\
\hline KT11 & 582408.714 & 2327958.001 & 7.346 & 582408.713 & 2327958.020 & 7.353 & -0.001 & 0.019 & 0.007 \\
\hline $\mathrm{KT} 16$ & 582407.854 & 2327956.836 & 7.020 & 582407.837 & 2327956.853 & 7.048 & -0.017 & 0.017 & 0.028 \\
\hline KT17 & 582407.682 & 2327955.162 & 7.234 & 582407.704 & 2327955.197 & 7.252 & 0.022 & 0.035 & 0.018 \\
\hline $\mathrm{KT} 27$ & 582407.672 & 2327954.543 & 6.961 & 582407.704 & 2327954.604 & 6.959 & 0.032 & 0.061 & -0.002 \\
\hline $\mathrm{KT} 18$ & 582407.684 & 2327954.119 & 7.231 & 582407.701 & 2327954.156 & 7.254 & 0.017 & 0.037 & 0.023 \\
\hline $\mathrm{KT} 36$ & 582407.707 & 2327944.315 & 7.202 & 582407.737 & 2327944.376 & 7.262 & 0.030 & 0.061 & 0.060 \\
\hline KT50 & 582408.896 & 2327927.338 & 7.264 & 582408.877 & 2327927.352 & 7.337 & -0.019 & 0.014 & 0.073 \\
\hline KT56 & 582408.874 & 2327920.882 & 7.360 & 582408.876 & 2327920.824 & 7.419 & 0.002 & -0.058 & 0.059 \\
\hline KT21 & 582407.673 & 2327952.309 & 6.945 & 582407.712 & 2327952.353 & 6.953 & 0.039 & 0.044 & 0.008 \\
\hline $\mathrm{KT} 25$ & 582407.668 & 2327951.683 & 7.247 & 582407.709 & 2327951.751 & 7.254 & 0.041 & 0.068 & 0.007 \\
\hline $\mathrm{KT} 26$ & 582407.662 & 2327951.194 & 6.913 & 582407.717 & 2327951.296 & 6.950 & 0.055 & 0.102 & 0.037 \\
\hline $\mathrm{KT} 28$ & 582407.652 & 2327950.339 & 7.219 & 582407.711 & 2327950.389 & 7.255 & 0.059 & 0.050 & 0.036 \\
\hline $\mathrm{KT} 29$ & 582407.683 & 2327949.921 & 6.959 & 582407.719 & 2327949.950 & 6.957 & 0.036 & 0.029 & -0.002 \\
\hline $\mathrm{KT} 30$ & 582407.665 & 2327949.056 & 7.195 & 582407.716 & 2327949.189 & 7.258 & 0.051 & 0.133 & 0.063 \\
\hline KT31 & 582407.692 & 2327948.392 & 6.921 & 582407.718 & 2327948.446 & 6.956 & 0.026 & 0.054 & 0.035 \\
\hline KT32 & 582407.671 & 2327947.634 & 7.230 & 582407.716 & 2327947.685 & 7.262 & 0.045 & 0.051 & 0.032 \\
\hline $\mathrm{KT} 33$ & 582407.692 & 2327947.032 & 6.897 & 582407.726 & 2327947.085 & 6.955 & 0.034 & 0.053 & 0.058 \\
\hline $\mathrm{KT} 34$ & 582407.706 & 2327946.129 & 7.200 & 582407.724 & 2327946.180 & 7.261 & 0.018 & 0.051 & 0.061 \\
\hline KT35 & 582407.703 & 2327945.366 & 6.924 & 582407.737 & 2327945.436 & 6.960 & 0.034 & 0.070 & 0.036 \\
\hline KT37 & 582407.730 & 2327943.308 & 6.933 & 582407.748 & 2327943.328 & 63 & 018 & 020 & 0.030 \\
\hline KT38 & 582407.746 & 2327942.209 & 7.231 & 582407.745 & 2327942.265 & 65 & -0.001 & .056 & 0.034 \\
\hline КT39 & 582407.748 & 2327941.427 & 6.917 & 582407.754 & 2327941.520 & 6.962 & 0.006 & 0.093 & 0.045 \\
\hline $\mathrm{KT} 40$ & 582407.770 & 2327940.362 & 7.216 & 582407.751 & 2327940.453 & 7.267 & -0.019 & 0.091 & 0.051 \\
\hline KT41 & 582407.726 & 2327939.353 & 6.906 & 582407.761 & 2327939.406 & 6.964 & 0.035 & 0.053 & 0.058 \\
\hline $\mathrm{K} T 42$ & 582407.808 & 2327938.017 & 7.188 & 582407.763 & 2327938.046 & 7.270 & -0.045 & 0.029 & 0.082 \\
\hline KT43 & 582407.748 & 2327936.974 & 6.927 & 582407.770 & 2327936.999 & 6.972 & 0.022 & 0.025 & 0.045 \\
\hline KT44 & 582407.713 & 2327935.559 & 7.215 & 582407.776 & 2327935.634 & 7.278 & 0.063 & 0.075 & 0.063 \\
\hline $\mathrm{KT} 45$ & 582407.710 & 2327934.475 & 6.917 & 582407.793 & 2327934.583 & 6.971 & 0.083 & 0.108 & 0.054 \\
\hline $\mathrm{KT} 46$ & 582407.779 & 2327933.652 & 7.204 & 582407.795 & 2327933.679 & 7.274 & 0.016 & 0.027 & 0.070 \\
\hline KT57 & 582408.701 & 2327932.971 & 7.200 & 582408.705 & 2327933.015 & 7.278 & 0.004 & 0.044 & 0.078 \\
\hline KT47 & 582408.903 & 2327931.325 & 7.221 & 582408.887 & 2327931.399 & 7.352 & -0.016 & 0.074 & 0.131 \\
\hline $\mathrm{KT} 48$ & 582408.796 & 2327929.819 & 7.304 & 582408.881 & 2327929.834 & 7.369 & 0.085 & 0.015 & 0.065 \\
\hline KT49 & 582408.773 & 2327928.679 & 7.275 & 582408.875 & 2327928.756 & 7.358 & 0.102 & 0.077 & 0.083 \\
\hline KT51 & 582408.778 & 2327926.205 & 7.277 & 582408.871 & 2327926.211 & 7.361 & 0.093 & 0.006 & 0.084 \\
\hline KT52 & 582408.864 & 2327924.879 & 7.279 & 582408.879 & 2327924.873 & 7.381 & 0.015 & -0.006 & 0.102 \\
\hline KT53 & 582408.787 & 2327923.788 & 7.298 & 582408.879 & 2327923.803 & 7.385 & 0.092 & 0.015 & 0.087 \\
\hline KT54 & 582408.876 & 2327922.403 & 7.305 & 582408.877 & 2327922.395 & 7.389 & 0.001 & -0.008 & 0.084 \\
\hline
\end{tabular}


geoscience application. Journal of Geophysical Research, v.117, F03017.

[2]. Yahya Alshawabkeh, Norbert Haala, 2006, Laser scanning and photogrammetry: a hybrid approach for heritage documentation. Conference: VAST 2006: The 7th International Symposium on Virtual Reality, Archaeology and Intelligent Cultural Heritage, Nicosia, Cyprus, 2006. Proceedings, 163-170.
[3]. P. Rönnholm, E. Honkavaara, P. Litkey, H. Hyyppä, J. Hyyppä., 2007, Intergration of laser scaning and photogrammetry, IAPRS Volume XXXVI, Part 3, 355-362.

[4]. M.J. Westoby, J. Brasington, F.F.Glasser, 2012, M.J. Hambrey, J.M. Reynolds, Structurefrom-Motion' photogrammetry: A low-cost, effective tool for geoscience application, Geomorphology 179, 300-314. O

\section{Summary}

Designing and manufacturing the Terrestrial Camera system based on photogrammetry technology that acquires geographic information data and create topographic maps

Dang Xuan Thuy, Luu Hai Au, Ngo Thi Lien

Vietnam Intitude of Geodesy and Cartography

Luu Hai Bang

Institute of Transport Science and Technology

Nowaday, the application of photogrammetry technology in collecting geographic information data and making topographic maps has become popular in the world and in Vietnam base on the advantages of this technology such as reducing time construction, reducing labor therefore bringing high economic benefits. The photogrammetry technology is assessed that it has great applicability, but it often has to be combined with direct measurement in the process of collecting geographic information data and creating topographic maps due to limitations that cannot be engraved in practice such as: lack of topographic and terrain data in high coverage areas, limited vertical data collection of the terrain... Therefore, in order to complete the process of applying photogrammetry technology in collecting geographic information data and creating topographic maps, studies are needed to overcome the above disadvantages. The authors researched based on the application of photogrammetry technology to overcome the above disadvantages. This paper introduces the Terrestrial Camera system based on photogrammetry technology that integrated by a digital camera, a GNSS and an IMU device. Besides, the paper investigates the accuracy of this system in collecting geographic information data and creating topographic maps. $\bigcirc$ 